\title{
Clinicopathologic Features, Treatment Response, and Outcomes of Immune Checkpoint Inhibitor-Related Esophagitis
}

\author{
Kavea Panneerselvam, MD${ }^{1}$; Rajan N. Amin, $\mathrm{MD}^{2}$; Dongguang Wei, MD³; Dongfeng Tan, $\mathrm{MD}^{3}$; Phillip J. Lum, BBA ${ }^{4}$;

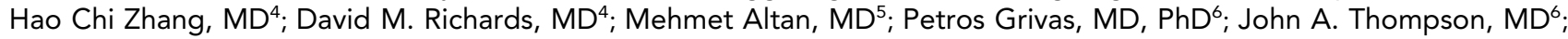 \\ Anusha $\mathrm{S}$. Thomas, $\mathrm{MD}^{4,}{ }^{*}$; and Yinghong Wang, $\mathrm{MD}, \mathrm{PhD}^{4, *}$
}

\begin{abstract}
Background: Although immune checkpoint inhibitors (ICls) have provided practice-changing outcomes in treating many cancers, $\mathrm{ICl}$-related gastrointestinal toxicity can limit their use. Upper gastrointestinal toxicity is not common nor as well described as lower gastrointestinal toxicity. We aimed to characterize the clinical presentation, endoscopic and histologic features, treatment response, and outcomes of $\mathrm{ICl}$-related esophagitis. Methods: We retrospectively studied patients at The University of Texas MD Anderson Cancer Center in whom esophagitis developed after receiving $\mathrm{ICls}$ from June 2011 through January 2020. We included patients with endoscopic evidence of esophagitis and excluded those with other obvious causes of esophagitis. A chi-square test was used to assess associations between categorical variables. The Mann-Whitney $U$ test was used to compare differences between continuous variables. Results: Of 657 consecutive patients who underwent esophagogastroduodenoscopy (EGD) during or within 6 months of completing ICl-based therapy, $21(3 \%)$ had esophagitis deemed to be from ICls. Of these patients, $1(5 \%)$ received an inhibitor of CTLA-4 alone, 15 (71\%) received anti-PD-1 or PD-L1 monotherapy, and 5 (24\%) received a combination of these. Median time from $\mathrm{ICl}$ initiation to onset of esophagitis was 4 months. Upon evaluation with EGD, only 3 patients $(14 \%)$ had isolated esophageal involvement; 18 (86\%) had concurrent involvement of the stomach, duodenum, or both. Most patients $(67 \%)$ were treated with proton pump inhibitors, and 4 (19\%) received steroids (prednisone or budesonide). The mortality rate was $38 \%$ (median follow-up, 15 months). Conclusions: Esophagitis associated with $\mathrm{ICl}$ use is rare. The diagnosis is one of exclusion because its clinical presentation appears similar to that of inflammation resulting from other causes. It often occurs in conjunction with other upper gastrointestinal toxicity. Symptoms are mild and respond well to nonimmunosuppressive treatment, with few severe complications.
\end{abstract}

J Natl Compr Canc Netw 2021;19(8):896-904 doi: $10.6004 /$ jnccn.2020.7675

\footnotetext{
${ }^{1}$ Department of Internal Medicine, Baylor College of Medicine, Houston, Texas; ${ }^{2}$ Department of Internal Medicine, University of Texas Health Science Center at Houston, Houston, Texas; and ${ }^{3}$ Department of Pathology, ${ }^{4}$ Department of Gastroenterology, Hepatology, and Nutrition, and ${ }^{5}$ Department of Thoracic/ Head \& Neck Medical Oncology, The University of Texas MD Anderson Cancer Center, Houston, Texas; and ${ }^{6}$ Department of Medicine, University of Washington, Fred Hutchinson Cancer Research Center, Seattle, Washington.

*These authors contributed equally as senior authors.
}

\section{Background}

Immune checkpoint inhibitors (ICIs) are revolutionary agents that are increasingly used to treat a variety of malignancies. ${ }^{1-3}$ These agents typically target inhibitory regulators of T cells, including CTLA-4, PD-1, and PD-L1. Blockade of these immune checkpoints leads to enhanced immune response against neoplastic cells. ${ }^{4,5}$ This has resulted in improved survival for multiple cancers. ${ }^{1-3}$

ICI use is associated with increased nonspecific immune response, which can result in toxic effects referred to as immune-related adverse events (irAEs). Commonly affected organs and organ systems include the gastrointestinal tract, skin, liver, lungs, and endocrine system. ${ }^{6-9}$ Gastrointestinal irAEs frequently present with lower gastrointestinal manifestations, such as diarrhea and colitis, and tend to be more common with CTLA-4 blockade than with PD-1 or PD-L1 blockade. ${ }^{10-12}$ In patients given CTLA- 4 inhibitors, reported incidence rates for grade 3/4 diarrhea and colitis were $18 \%$ and $5 \%$, respectively, ${ }^{13,14}$ with average times to symptom onset ranging from 6 to 9 weeks. ${ }^{14,15}$

Upper gastrointestinal tract irAEs are less common than lower gastrointestinal irAEs, and the clinical manifestations of upper gastrointestinal toxicity are less well

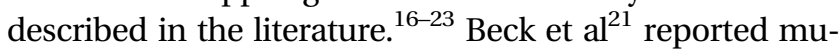
cosal injury limited to the stomach and duodenum in 5\% of patients treated with ICIs. Additionally, Tang et $\mathrm{al}^{18}$ found that upper gastrointestinal irAEs were more commonly associated with anti-PD-1/PD-L1 agents than with anti-CTLA-4 agents, with gastric involvement more common than duodenal involvement according to endoscopy and histology. Similar to other upper gastrointestinal irAEs, these episodes of esophagitis were associated with use of anti-PD-1 agents. ${ }^{16,17,24,25}$ Onset of upper gastrointestinal irAEs in these cases is later than that of lower gastrointestinal irAEs, with Boike and Dejulio ${ }^{16}$ and Onuki et $\mathrm{al}^{17}$ describing onset of symptoms 6 months after initiation of treatment with anti-PD-1 agents. Other than these few cases, there has been little published literature describing the endoscopic and histologic features of ICI-related esophagitis, treatment response, or clinical outcomes. 
There is a gap in knowledge regarding the typical characteristics of ICI-related esophagitis and the appropriate treatments for this condition. In this retrospective study, we aimed to characterize esophagitis associated with the use of ICIs and describe its clinical, endoscopic, and histologic features. Additionally, we sought to determine the severity, disease course, treatment response, and outcomes of esophagitis.

\section{Methods}

\section{Study Design}

This retrospective analysis included consecutive patients at The University of Texas MD Anderson Cancer Center (MDACC) receiving ICI-based therapy from June 2011 to January 2020 who underwent upper endoscopies revealing esophagitis. Patients with preexisting gastroesophageal reflux disease (GERD), eosinophilic esophagitis, esophageal cancer, or known esophageal infections (eg, Candida, cytomegalovirus, herpes simplex virus) or who underwent esophagogastric surgery or head, neck, or chest irradiation were excluded. In the included patients, esophagitis was determined to be caused by ICIs based on endoscopic appearance and the exclusion of other causes of esophagitis.

This study was approved by the Institutional Review Board of MDACC (PA18-0472). Consent was waived for this study and a waiver of consent was granted.

\section{Patient Characteristics}

The following information was obtained via review of the patients' medical records: demographic variables (eg, age, sex, race), smoking status, medical comorbidities (eg, hypertension, hyperlipidemia, diabetes mellitus), cancer type and stage according to the 7th edition of the AJCC Cancer Staging Manual, type and duration of ICIbased therapy, chemotherapy and nonsteroidal anti-inflammatory drug (NSAID) use within 3 months of ICI use, and other reported irAEs.

\section{Clinical Characteristics of Esophagitis}

The following esophagitis-related information was collected from the patients' medical records: presenting symptoms, indications for esophagogastroduodenoscopy (EGD), grade of esophagitis using the CTCAE, severity of esophagitis (Los Angeles Classification System grade), anatomic location and pattern of esophagitis, time from ICI treatment initiation to onset of toxicity, and histologic diagnosis.

The patients' EGD reports and pathologic images and reports were reviewed. The distribution of esophageal inflammation was divided into the upper, middle, and distal thirds of the esophagus, and the gastroesophageal junction. The pattern of esophagitis was described as patchy or diffuse based on the assessment of 2 endoscopists. The histologic findings were categorized as acute inflammation (neutrophilic or eosinophilic infiltration, fibrinous exudates, or ulcerations) or chronic inflammation (histiocytic, plasma cell, or lymphocytic infiltration; fibrosis; or architecture distortion) assessed by 2 gastrointestinal pathologists. Patients with features of both acute and chronic inflammation were included in the chronic inflammation category.

Medical records were also reviewed for treatment with proton pump inhibitors (PPIs), sucralfate, histamine 2 receptor $\left(\mathrm{H}_{2}\right)$ blockers, and steroids. Immunosuppressant use for esophagitis and for other gastrointestinal toxic effects was also recorded. Hospitalization, length of hospital stay, complications related to esophagitis, recurrence of esophagitis, and data from follow-up endoscopy were also gathered. The date of last follow-up visit or date of death for each patient was also collected.

\section{Statistical Analysis}

Categorical variables were summarized using frequencies and percentages. Continuous variables were summarized using means and standard deviations or medians and interquartile ranges (IQRs). A chi-square test was used to analyze associations between categorical variables. The Mann-Whitney $U$ test was used to compare differences between continuous variables. $P$ values $<.05$ were considered significant.

\section{Results}

\section{Patient Characteristics}

Of 657 patients who received ICI-based therapy and underwent EGD at MDACC during the study period, 21 (3\%) had esophagitis deemed to be associated with ICI use and not attributable to any other obvious etiology.

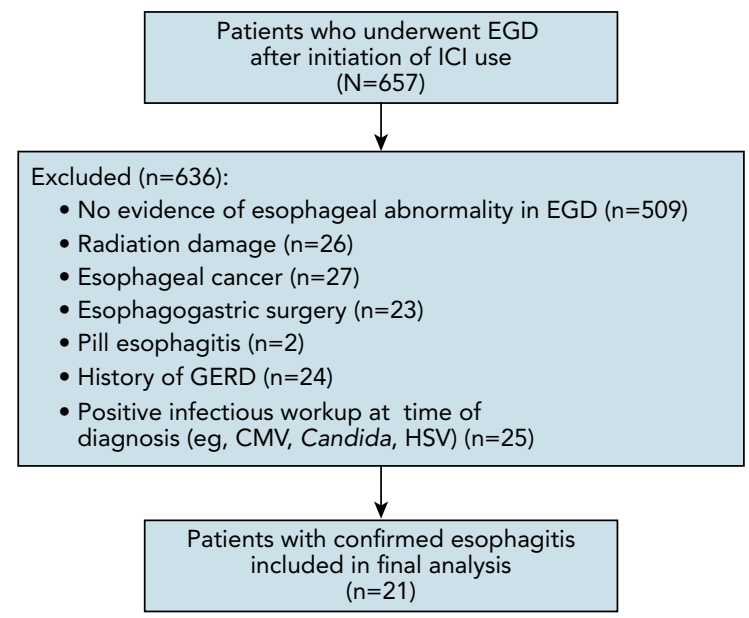

Figure 1. Selection flowchart of the study patients. Abbreviations: CMV, cytomegalovirus; EGD, esophagogastroduodenoscopy; GERD, gastroesophageal reflux disease; HSV, herpes simplex virus; ICI, immune checkpoint inhibitor. 


\section{Table 1. Patient Characteristics}

\begin{tabular}{|lc|}
\hline Characteristic & $\mathrm{n}(\%)$ \\
\hline Total, N & 21 \\
\hline Mean age (SD), y & $64(10)$ \\
\hline Male sex & $13(62)$ \\
\hline Race/Ethnicity & $13(62)$ \\
\hline White & $4(19)$ \\
\hline Black & $3(14)$ \\
\hline Hispanic & $1(5)$ \\
\hline Other & $11(52)$ \\
\hline Ever-smoker & \\
\hline Comorbidities & $6(29)$ \\
\hline Hypertension & $6(29)$ \\
\hline Hyperlipidemia & $5(24)$ \\
\hline Diabetes mellitus, type 2 & $5(24)$ \\
\hline Hypothyroidism & $3(14)$ \\
\hline Chronic kidney disease & $2(10)$ \\
\hline Ischemic heart disease & $2(10)$ \\
\hline COPD & $2(10)$ \\
\hline Cancer type & $1(5)$ \\
\hline NSCLC & $1(5)$ \\
\hline Gastrointestinal & $4(19)$ \\
\hline Melanoma & $3(14)$ \\
\hline Henitourinary & $29)$ \\
\hline Hematologic & \\
\hline Other & \\
\hline
\end{tabular}

(continued)

The patient selection diagram is shown in Figure 1. We observed that in this small cohort, 1 patient $(5 \%)$ received anti-CTLA-4 monotherapy, 15 (71\%) received anti-PD-1/ PD-L1 monotherapy, and 5 (24\%) received a combination of these. Four patients (19\%) received chemotherapy known to rarely cause esophagitis concurrently with ICI therapy, namely pemetrexed, docetaxel, or cytarabine. ${ }^{26-28}$ Only 1 patient (5\%) took NSAIDs within 3 months before the onset of esophagitis.

Patient characteristics are listed in Table 1. Mean patient age at the time of ICI initiation was 64 years. Most patients were White $(62 \%)$ and male $(62 \%)$. Approximately one-half of the patients $(52 \%)$ had a history of smoking, and $43 \%$ had comorbidities, including hypertension (29\%), hyperlipidemia (29\%), type 2 diabetes mellitus (24\%), and hypothyroidism (24\%). The most common malignancy was non-small cell lung cancer $(n=6 ; 29 \%)$ followed by cancer of the gastrointestinal tract $(n=4 ; 19 \%)$ and melanoma $(\mathrm{n}=4 ; 19 \%)$. The most common ICI-related toxic effect outside of the upper gastrointestinal system was colitis (24\%).

\begin{tabular}{|lc|}
\hline \multicolumn{2}{|l|}{ Table 1. Patient Characteristics (cont.) } \\
\hline Characteristic & $\mathbf{n}(\%)$ \\
\hline AJCC cancer stage ${ }^{\text {b }}$ & $1(5)$ \\
\hline III & $19(95)$ \\
\hline ICl type & $1(5)$ \\
\hline Anti-CTLA-4 & $15(71)$ \\
\hline $\begin{array}{l}\text { Anti-PD-1/PD-L1 } \\
\text { Combination }\end{array}$ & $5(24)$ \\
\hline $\begin{array}{l}\text { ICl given with chemotherapy known to } \\
\text { cause esophagitis }\end{array}$ & $4(19)$ \\
\hline ICl given concurrently with any \\
chemotherapy
\end{tabular}

Abbreviations: COPD, chronic obstructive pulmonary disease; $\mathrm{ICl}$, immune checkpoint inhibitor; irAE, immune-related adverse effect; NSAID, nonsteroidal anti-inflammatory drug; NSCLC, non-small cell lung cancer.

${ }^{a}$ Cancer of the orbit.

${ }^{\mathrm{b}}$ Available for 20 patients with solid tumors or melanoma.

'2 patients received pemetrexed, 1 received docetaxel, and 1 received cytarabine.

Clinical Characteristics and Treatment of Esophagitis Patients underwent EGD primarily for evaluation of nausea and vomiting (33\%), dysphagia (19\%), or hematemesis (19\%). The median time from ICI initiation to onset of endoscopic evidence of esophagitis was 4 months (interquartile range [IQR], 2-9 months), with a median of 3 ICI infusions (IQR, 1.5-5.5). The median duration of esophagitis symptoms was 30 days (IQR, 9-43 days). Most patients $(\mathrm{n}=17 ; 81 \%)$ had mild esophagitis (CTCAE grade 1-2) (Table 2).

Additional endoscopic and histologic findings are summarized in Table 3 . Esophagitis severity varied widely from mild inflammation to severe inflammation with ulceration based on endoscopy (Figure 2). On EGD, only 3 patients (14\%) had isolated esophageal involvement; 18 (86\%) had concurrent involvement of the stomach, duodenum, or both. A total of 11 patients $(52 \%)$ had inflammation of multiple segments of the esophagus, whereas the others had isolated involvement of the middle one-third of the esophagus $(n=7 ; 33 \%)$, distal third of the esophagus $(n=2 ; 10 \%)$, or gastroesophageal junction $(\mathrm{n}=1 ; 5 \%)$. Only 10 patients (48\%) underwent esophageal biopsies; 5 demonstrated acute inflammation, whereas 
Table 2. Clinical Information Related to Treatment With ICls and Esophagitis $(\mathrm{N}=21)$

\begin{tabular}{|c|c|}
\hline Characteristic & $\mathrm{n}(\%)$ \\
\hline Median duration of ICl-based therapy (IOR), $d$ & $105(49-433)$ \\
\hline $\begin{array}{l}\text { Median number of } \mathrm{ICl} \text { infusions at time } \\
\text { of esophagitis onset (IQR) }\end{array}$ & $3(1.5-5.5)$ \\
\hline $\begin{array}{l}\text { Median time from initiation of } \mathrm{ICl} \text { to onset of } \\
\text { esophagitis (IQR), } d\end{array}$ & $126(62-272)$ \\
\hline Median duration of symptoms (IQR), $d$ & $30(9-43)$ \\
\hline \multicolumn{2}{|l|}{ Clinical esophagitis presentations } \\
\hline Nausea/Vomiting & $14(67)$ \\
\hline Dysphagia/Odynophagia & $6(29)$ \\
\hline Hematemesis & $4(19)$ \\
\hline Melena & $3(14)$ \\
\hline Dyspepsia & $2(10)$ \\
\hline Reflux & $1(5)$ \\
\hline None (incidental finding) ${ }^{a}$ & $4(19)$ \\
\hline \multicolumn{2}{|l|}{ EGD indications } \\
\hline Nausea/Vomiting & $7(33)$ \\
\hline Dysphagia & $4(19)$ \\
\hline Hematemesis & $4(19)$ \\
\hline Anemia & $2(10)$ \\
\hline Abdominal pain & $1(5)$ \\
\hline Melena & $1(5)$ \\
\hline Reflux & $1(5)$ \\
\hline PEG placement & $1(5)$ \\
\hline \multicolumn{2}{|l|}{ Peak CTCAE grade of esophagitis } \\
\hline $1-2$ & $17(81)$ \\
\hline $3-4$ & $4(19)$ \\
\hline \multicolumn{2}{|l|}{ Los Angeles grade of esophagitis } \\
\hline$A$ & $3(14)$ \\
\hline B & 4 (19) \\
\hline C & $2(10)$ \\
\hline$D$ & $5(24)$ \\
\hline Unspecified & $7(33)$ \\
\hline
\end{tabular}

Abbreviations: EGD, esophagogastroduodenoscopy; $\mathrm{ICl}$, immune checkpoint inhibitor; IQR, interquartile range; PEG, percutaneous endoscopic gastrostomy.

a 2 EGDs performed for iron deficiency anemia, 1 for lower abdominal pain, and 1 for PEG placement.

the other 5 demonstrated chronic inflammation. Of the 11 patients who did not undergo biopsy, one had a prior EGD that showed a normal esophagus and 10 had no prior EGDs.

The treatments and outcomes of esophagitis are listed in Table 4 . A total of 10 patients (48\%) required hospitalization for upper gastrointestinal symptoms. Patients most commonly received PPI treatment $(n=14 ; 67 \%)$; only 4 (19\%) received steroids (3 received prednisone and
Table 3. Endoscopic and Pathologic Findings

\begin{tabular}{|c|c|}
\hline Characteristic & n (\%) \\
\hline Total, N & 21 \\
\hline \multicolumn{2}{|l|}{ EGD findings } \\
\hline \multicolumn{2}{|l|}{ Esophagus } \\
\hline Ulcer & $4(19)$ \\
\hline Nonulcer inflammation & $17(81)$ \\
\hline \multicolumn{2}{|l|}{ Nonesophagus } \\
\hline Gastritis/Gastric ulcer & $17(81)$ \\
\hline Duodenitis & $8(38)$ \\
\hline Isolated esophageal involvement & $3(14)$ \\
\hline Concurrent esophageal and gastric involvement & $10(48)$ \\
\hline Concurrent esophageal and duodenal involvement & $1(5)$ \\
\hline $\begin{array}{l}\text { Concurrent esophageal, gastric, and duodenal } \\
\text { involvement }\end{array}$ & $7(33)$ \\
\hline \multicolumn{2}{|l|}{ Esophagitis distribution } \\
\hline Top one-third only & 0 \\
\hline Middle one-third only & $7(33)$ \\
\hline Distal one-third only & $2(10)$ \\
\hline GEJ only & $1(5)$ \\
\hline Multilocation involvement & $11(52)$ \\
\hline \multicolumn{2}{|l|}{ Esophagitis distribution pattern } \\
\hline Patchy & $15(71)$ \\
\hline Diffuse & $6(29)$ \\
\hline \multicolumn{2}{|l|}{ Histologic features $(n=10)$} \\
\hline Acute inflammation & $5(50)$ \\
\hline Chronic active inflammation & $5(50)$ \\
\hline
\end{tabular}

Abbreviations: EGD, esophagogastroduodenoscopy; GEJ, gastroesophageal junction.

1 received oral budesonide) for a median duration of 50 days (IQR, 35-134 days). Infliximab and vedolizumab were administered for concurrent lower gastrointestinal toxic effect. Of 17 symptomatic patients, 15 experienced symptom resolution after the initial course of treatment with PPIs, steroids, sucralfate, $\mathrm{H}_{2}$ blockers, or a combination of these. Two had recurrent symptoms after initially receiving treatment with PPIs: 1 after 12 days and the other after 182 days. Afterward, one of these patients received treatment with steroids, and the other received a PPI, resulting in complete resolution of their symptoms. The remaining 2 patients died due to other causes and never experienced symptom resolution. Of the 5 patients who had a repeat EGD, 4 had resolution of esophagitis.

Serious complications caused by esophagitis were rare; 3 patients (14\%) had bleeding upon EGD and $1(5 \%)$ required total parenteral nutrition support. ICI-based therapy was stopped permanently for 15 patients because of all irAEs. Esophagitis was the principal toxic effect leading to discontinuation of ICI-based therapy in 

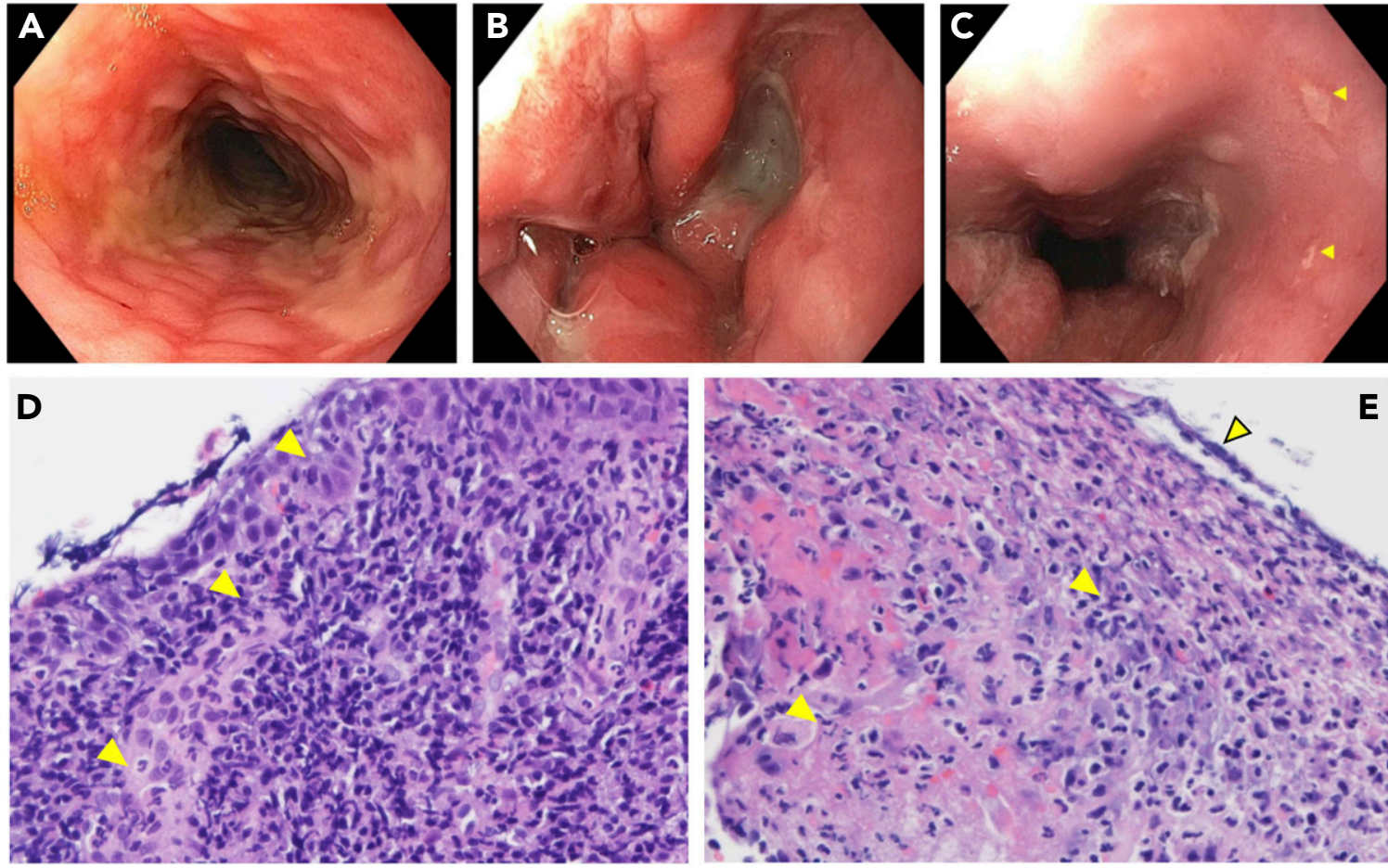

Figure 2. Endoscopic and histologic features of $\mathrm{ICl}$-related esophagitis. (A) Multiple large circumferential ulcers in the middle third of the esophagus. (B) Inflammation in the GEJ. (C) Nonbleeding erosions in the lower third of the esophagus. (D) Hematoxylin-eosin-stained section (original magnification $\times 20$ ) of esophageal mucosa showing chronic active esophagitis, with increased numbers of neutrophils in the squamous epithelium and lamina propria. Granulation tissue, clusters of lymphocytes, and plasma cells are shown in the lamina propria (arrows). (E) Hematoxylin-eosin-stained section (original magnification $\times 20$ ) of esophageal mucosa showing esophageal ulceration with underlying chronic active inflammation (arrows).

Abbreviations: GEJ, gastroesophageal junction; ICl, immune checkpoint inhibitor.

only 2 patients. A total of 8 patients (38\%) died during the study period (median follow-up, 15 months [IQR, 4 months to 4.2 years]); all were attributed to the patients' malignancies.

\section{Patient Characteristics According to \\ Esophagitis Risk Factors}

We compared the clinical characteristics of patients with risk factors for esophagitis other than ICI use and those without other risk factors. We defined these risk factors as NSAID use within 3 months prior to esophagitis onset, any chemotherapy administered within 3 months prior to esophagitis onset, and chemotherapy given concurrently with ICIs. We found no statistically significant differences in the features of inflammation $(P=.92)$, esophagitis distribution $(P=.36)$, duration from initiation of ICI use to onset of esophagitis $(P=.34)$, or duration of hospitalization $(P=.71)$ or symptoms $(P=.77)$ between these 2 groups (Table 5).

\section{Discussion}

Gastrointestinal toxicity is one of the most common types of toxicity, occurring in up to $35 \%$ of patients treated with ICIs. ${ }^{29-31}$ Although gastrointestinal irAEs can affect any segment of the gastrointestinal tract, they tend to be most common in the colon. Most trials in patients with gastrointestinal toxic effects of ICIs have mainly focused on colitis. ${ }^{11,32,33}$ Tang et $\mathrm{al}^{18}$ reported on the largest case series of upper gastrointestinal irAEs in 2019, which included 60 patients. ICI-related esophagitis in particular is exceptionally rare, with only 4 cases reported in the literature. ${ }^{16,17,34,35}$ Our report represents the largest case series of esophagitis attributed to the use of ICIs.

The spectrum of ICI-related esophagitis clinical presentations is wide, and endoscopic features range from mild inflammation to severe ulceration. ${ }^{16,18,34}$ Most of our patients had low-grade esophagitis symptoms, with $81 \%$ experiencing peak CTCAE grades of 1 or 2 . Most of the patients had symptom resolution with simple, low-cost treatment, with only 2 having recurrent symptoms. These treatments included PPIs, $\mathrm{H}_{2}$ blockers, and sucralfate. Only 4 patients received steroids in the context of concurrent colitis, and none of these 4 received other immunosuppressants, such as infliximab and vedolizumab. Overall, esophagitis tended to be mild, and symptoms resolved quickly without the need for aggressive immunosuppression. Systemic steroids seemed 
Table 4. Treatment and Outcomes of Esophagitis

\begin{tabular}{|c|c|}
\hline Characteristic & $\mathrm{n}(\%)$ \\
\hline Total, N & 21 \\
\hline Hospitalization & $10(48)$ \\
\hline Esophagitis diagnosed during hospitalization & $3(14)$ \\
\hline Median duration of hospitalization (IQR), d ( $n=10)$ & $7(4.5-9.5)$ \\
\hline \multicolumn{2}{|l|}{ Treatment } \\
\hline PPI & $14(67)$ \\
\hline Sucralfate & $4(19)$ \\
\hline $\mathrm{H}_{2}$ blocker & $2(10)$ \\
\hline Steroids ${ }^{a}$ & $4(19)$ \\
\hline No treatment & $5(24)$ \\
\hline $\begin{array}{l}\text { Median duration of steroid-based } \\
\text { treatment (IQR), } d(n=4)\end{array}$ & $50(35-134)$ \\
\hline \multicolumn{2}{|c|}{ Immunosuppressant use for coexisting gastrointestinal toxic effects } \\
\hline Steroids & $2(10)$ \\
\hline Vedolizumab ${ }^{b}$ & $3(14)$ \\
\hline \multicolumn{2}{|l|}{ Complications related to esophagitis } \\
\hline Bleeding & $3(14)$ \\
\hline $\begin{array}{l}\text { Feeding difficulty requiring total } \\
\text { parenteral nutrition support }\end{array}$ & $1(5)$ \\
\hline \multicolumn{2}{|l|}{$\mathrm{ICl}$ use stopped } \\
\hline Because of gastrointestinal toxicity ${ }^{d}$ & $9(43)$ \\
\hline Because of other reason & $6(29)$ \\
\hline Recurrent esophagitis symptoms & $2(10)$ \\
\hline Repeat EGD performed & $5(24)$ \\
\hline Resolution of esophagitis upon repeat EGD $(n=5)$ & $4(80)$ \\
\hline $\begin{array}{l}\text { Median duration from first to } \\
\text { repeat EGD (IQR), } d(n=5)\end{array}$ & $59(39-179)$ \\
\hline Median follow-up duration (IQR), d ( $n=21)$ & $464(271-820)$ \\
\hline Death resulting from any cause & $8(38)$ \\
\hline Death caused by malignancy & $8(38)$ \\
\hline
\end{tabular}

Abbreviations: EGD, esophagogastroduodenoscopy; $\mathrm{H}_{2}$, histamine 2 receptor; ICl, immune checkpoint inhibitor; IQR, interquartile range; PPI, proton pump inhibitor.

a 3 patients received prednisone and 1 received oral budesonide.

bUsed for ICl-induced gastritis and colitis.

${ }^{\mathrm{c}}$ Total parenteral nutrition used because of persistently bleeding esophagitis.

${ }^{\mathrm{d}}$ All patients had concurrent esophageal and other gastrointestinal toxic effects.

to work well for severe cases. However, drawing strong conclusions on the utility of steroids for management of ICI-related esophagitis in this cohort is difficult given multiple confounding factors. The role of immunosuppression in treating ICI-related esophagitis will require larger prospective studies.

The reported upper gastrointestinal symptoms of ICI-related esophagitis were nonspecific. We excluded patients with a history of conditions that cause esophagitis, such as GERD, eosinophilic esophagitis, and known or suspected pill esophagitis. In addition, we examined characteristics such as distribution and histologic patterns to help distinguish different types of esophagitis. For example, pill esophagitis often occurs in the middle one-third of the esophagus, tends to be focal, and can present with neutrophilic inflammation. ${ }^{36}$ Reflux and eosinophilic esophagitis tend to have different endoscopic distributions, and although both can present with eosinophilia, it can vary in degree and location. ${ }^{36,37}$ In our cohort, $33 \%$ of the patients had esophagitis isolated to the middle one-third of the esophagus, and $52 \%$ had a diffuse distribution. The histopathology of the esophageal biopsy specimens in our study demonstrated features of both acute and chronic inflammation similar to those seen in ICI-related colitis. ${ }^{9}$ These findings are consistent with those of a recent study by Patil and Zhang ${ }^{38}$ that showed ICI-related esophagitis was characterized by ulcerations and lymphocytic inflammation. Given the lack of pathognomonic features of ICI-related esophagitis, a definitive diagnosis of ICI-related esophagitis could not be made based on histologic evaluation alone. ICI-related esophagitis remains a diagnosis of exclusion.

In our small cohort, we observed that anti-PD-1/PD-L1 therapies were more commonly associated with esophagitis than were anti-CTLA-4 therapies, which is consistent with previous reports. A meta-analysis by Khoja et $\mathrm{al}^{32}$ of 48 trials of ICI-based monotherapy demonstrated that colitis was strongly associated with anti-CTLA-4 therapy (odds ratio, 8.7; 95\% CI, 5.8-12.9). Although data on upper gastrointestinal toxicity are limited, the case series by Tang et $\mathrm{al}^{18}$ showed an association between treatment with PD-1/PD-L1 inhibitors and upper gastrointestinal toxicity. Case reports by Boike and Dejulio ${ }^{16}$ and Onuki et $\mathrm{al}^{17}$ also demonstrated upper gastrointestinal toxicity associated with treatment with the PD-1 inhibitors nivolumab and pembrolizumab, respectively. In addition, use of anti-CTLA-4 regimens has been associated with more severe toxicity than has use of anti-PD-1/PD-L1 regimens. ${ }^{31,39,40}$ Researchers hypothesized that the specific toxic effects associated with each class of ICI are related to variable expression of the targets in different tissues. ${ }^{32,41}$ However, the distribution of CTLA-4 and PD-1/PD-L1 expression along the gastrointestinal tract has not been well described.

The median time from initiation of ICI use to onset of esophagitis in our study was approximately 4 months, which is similar to the time of onset described by other authors. ${ }^{18}$ This is a longer time to onset than is generally observed for other irAEs. For example, lower gastrointestinal irAEs such as colitis tend to have an onset of 6 to 8 weeks, ${ }^{42}$ in contrast with upper gastrointestinal irAEs, which tend to have an onset of 4 to 9 months. ${ }^{16-18,43}$ However, retrospective analyses have shown that colitis associated with PD-1/PD-L1 blockade 


\begin{tabular}{|c|c|c|c|}
\hline Total, n & 10 & 11 & \\
\hline EGD findings & & & .92 \\
\hline Esophagitis distribution upon EGD & & & .36 \\
\hline Top one-third only & $0(0)$ & $0(0)$ & \\
\hline Middle one-third only & $3(30)$ & $4(36)$ & \\
\hline Distal one-third only & $0(0)$ & $2(18)$ & \\
\hline Median duration of hospitalization (IQR), d ( $n=13)$ & $8(5.5-9.0)$ & $6.5(2.8-9.5)$ & .71 \\
\hline Median duration of symptoms (IQR), d ( $n=15)$ & $28(11-47)$ & $30(8-51)$ & .77 \\
\hline
\end{tabular}

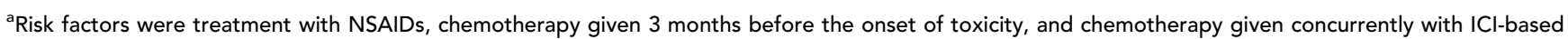
treatment.

Abbreviations: EGD, esophagogastroduodenoscopy; GEJ, gastroesophageal junction; ICl, immune checkpoint inhibitor; IQR, interquartile range; NSAID, nonsteroidal anti-inflammatory drug.

has a later onset than does colitis associated with CTLA4 blockade. $^{39}$ Delayed onset of upper gastrointestinal toxic effects may be explained by their association with anti-PD-1/PD-L1 therapy. Confirming this requires further evaluation.

In our cohort, $77 \%$ of the patients received PPIs or $\mathrm{H}_{2}$ blockers, and their symptoms resolved in a median of 30 days. In our median follow-up period of 15 months, we did not observe any long-term sequelae, such as esophageal stricture and Barrett esophagus. However, given the low percentage of repeat EGDs (24\%) and the short duration between serial EGDs (median, 59 days), our data are likely insufficient to describe long-term consequences of ICI-related esophagitis. A longer follow-up period with close surveillance would be needed to detect long-term complications of ICI-related esophagitis.

Patients with varying clinical grades of esophagitis, namely CTCAE grades 1 through 4 , had similar endoscopic findings. Therefore, it appeared that clinical grade does not correlate with endoscopic features of severity. However, this finding could be limited by our small sample size. Likewise, we also speculate that endoscopic features of esophagitis cannot be used as prognostic factors to determine the need for aggressive therapy based on our data. A larger-scale study would be needed to determine high-risk endoscopic features of esophagitis and its prognostic value. Because the prevalence of ICI-related esophagitis is low, we recommend initial empirical therapy with PPIs in the absence of alarming symptoms. If symptoms persist, endoscopy could be pursued along with additional therapies, such as steroids. Although evidence supporting this proposed management of ICI-related esophagitis is lacking, it is routinely used for esophagitis of other etiologies with reasonable success.

Despite the many strengths of our study, its limitations are important to acknowledge. First, data collection was relatively limited because of the retrospective, single-center study nature, which may have resulted in some selection and confounding biases. Second, some patients may have had mild symptoms that did not trigger endoscopy and were excluded from the study. Third, given a lack of current guidelines for treatment of ICI-related esophagitis, patients underwent treatment according to the treating physician's discretion, leading to variation in treatment. Fourth, the study lacked randomization, a control population, and biomarker assessment. Fifth, the lack of pathognomonic histologic and clinical features of ICI-related esophagitis makes it a diagnosis of exclusion. For example, cases that occurred in the context of underlying GERD or eosinophilic esophagitis could have been excluded. Inversely, lack of recognition of confounding conditions may have caused inclusion of cases that should have been excluded. Sixth, although this is the largest study of its kind, the small sample restricted advanced statistical analyses and comparisons. Despite these limitations, our study generated interesting hypotheses and can inform the sparse literature on this important topic. 


\section{Conclusions}

The rate of ICI-related esophagitis in patients with upper gastrointestinal symptoms undergoing EGD is as low as $3 \%$. It presents similarly to esophagitis of other etiologies and remains a diagnosis of exclusion. Esophageal toxic effects frequently present in conjunction with other gastrointestinal toxic effects that are more severe, but alone rarely lead to termination of ICI use. Most patients have mild symptoms, and their esophagitis can be effectively managed with nonimmunosuppressive treatments. The benefit of use of steroids is unclear. A larger prospective study with longer follow-up would help further characterize this condition longitudinally and determine optimal management.

\section{Acknowledgments}

The datasets used and analyzed in the present study are available from the corresponding author upon reasonable request. Editing of this paper was provided by Scientific Publications, Research Medical Library at The University of Texas MD Anderson Cancer Center.
Submitted September 5, 2020; final revision received October 18, 2020; accepted for publication October 20, 2020. Published online June 11, 2021.

Author contributions: Study concept and design: Thomas, Wang. Data acquisition: Panneerselvan, Lum. Data analysis and interpretation: Panneerselvan, Thomas, Wang. Manuscript preparation: Panneerselvam, Thomas, Wang. Critical revision: Amin, Zhang, Richards, Altan, Grivas, Thompson, Thomas, Wang. Pathology slide review and histologic images: Wei, Tan.

Disclosures: Dr. Grivas has disclosed serving as a consultant or in an advisory role for AstraZeneca, Bayer, Bristol Myers Squibb, Clovis Oncology, Dyania Health, Driver, EMD Serono, Exelixis, Foundation Medicine, Genentech/Roche, Genzyme, GlaxoSmithKline, Heron Therapeutics, Immunomedics, Infinity Pharmaceuticals, Janssen, Merck \& Co., Mirati Therapeutics, Pfizer, QED Therapeutics, and Seattle Genetics; and receiving research support from Bavarian Nordic, Bristol Myers Squibb, Clovis Oncology, Debiopharm, GlaxoSmithKline, Immunomedics, Kure It Cancer Research, Merck \& Co., Mirati Therapeutics, Pfizer, and QED Therapeutics. Dr. Thompson has disclosed receiving grant/research support from Merck, Hoffmann-LaRoche, Pfizer, Five Prime, Novartis, Incyte, Trillium, and Xencor; and serving as a consultant for Neoleukin, Aveo, and

Regeneron. Dr. Wang has disclosed serving as a consultant for Tillotts Pharma. The remaining authors have disclosed that they have not received any financial consideration from any person or organization to support the preparation, analysis, results, or discussion of this article.

Correspondence: Yinghong Wang, MD, PhD, Department of Gastroenterology, Hepatology and Nutrition, The University of Texas MD Anderson Cancer Center, Unit 1466, 1515 Holcombe Boulevard, Houston, TX 77030. Email: ywang59@mdanderson.org

\section{References}

1. Borghaei $H$, Paz-Ares $L$, Horn L, et al. Nivolumab versus docetaxel in advanced nonsquamous non-small-cell lung cancer. N Engl J Med 2015; 373:1627-1639.

2. Topalian SL, Sznol M, McDermott DF, et al. Survival, durable tumor remission, and long-term safety in patients with advanced melanoma receiving nivolumab. J Clin Oncol 2014;32:1020-1030.

3. Wolchok JD, Chiarion-Sileni V, Gonzalez R, et al. Overall survival with combined nivolumab and ipilimumab in advanced melanoma. $\mathrm{N}$ Engl $\mathrm{J}$ Med 2017;377:1345-1356.

4. Topalian SL, Drake CG, Pardoll DM. Immune checkpoint blockade: a common denominator approach to cancer therapy. Cancer Cell 2015;27 450-461.

5. Pardoll DM. The blockade of immune checkpoints in cancer immunotherapy. Nat Rev Cancer 2012;12:252-264.

6. Puzanov I, Diab A, Abdallah K, et al. Managing toxicities associated with immune checkpoint inhibitors: consensus recommendations from the Society for Immunotherapy of Cancer (SITC) Toxicity Management Working Group. J Immunother Cancer 2017;5:95.

7. Weber JS, Kähler KC, Hauschild A. Management of immune-related adverse events and kinetics of response with ipilimumab. J Clin Oncol 2012;30:2691-2697.

8. Michot JM, Bigenwald C, Champiat S, et al. Immune-related adverse events with immune checkpoint blockade: a comprehensive review. Eur J Cancer 2016;54:139-148.

9. Wang $Y$, Abu-Sbeih $H$, Mao E, et al. Endoscopic and histologic features of immune checkpoint inhibitor-related colitis. Inflamm Bowel Dis 2018; 24:1695-1705.

10. Kumar V, Chaudhary N, Garg M, et al. Current diagnosis and management of immune related adverse events (irAEs) induced by immune checkpoint inhibitor therapy. Front Pharmacol 2017;8:49.

11. Robert C, Schachter J, Long GV, et al. Pembrolizumab versus ipilimumab in advanced melanoma. N Engl J Med 2015;372:2521-2532.

12. Powles T, Eder JP, Fine GD, et al. MPDL3280A (anti-PD-L1) treatment leads to clinical activity in metastatic bladder cancer. Nature 2014;515: 558-562.

13. Robert C, Thomas L, Bondarenko I, et al. Ipilimumab plus dacarbazine for previously untreated metastatic melanoma. N Engl J Med 2011;364: 2517-2526.

14. Hodi FS, Postow MA, Chesney JA, et al. Clinical response, progressionfree survival (PFS), and safety in patients (pts) with advanced melanoma (MEL) receiving nivolumab (NIVO) combined with ipilimumab (IPI) vs IPI monotherapy in CheckMate 069 study [abstract]. J Clin Oncol 2015; 33(Suppl):Abstract 9004.

15. Spain L, Diem S, Larkin J. Management of toxicities of immune checkpoint inhibitors. Cancer Treat Rev 2016;44:51-60.

16. Boike J, Dejulio T. Severe esophagitis and gastritis from nivolumab therapy. ACG Case Rep J 2017;4:e57.

17. Onuki T, Morita E, Sakamoto N, et al. Severe upper gastrointestinal disorders in pembrolizumab-treated non-small cell lung cancer patient. Respirol Case Rep 2018;6:e00334.

19. Shi $Y$, Lin $P, H o E Y$, et al. Nivolumab-associated nausea and vomiting as an immune adverse event. Eur J Cancer 2017;84:367-369.

20. Gonzalez RS, Salaria SN, Bohannon CD, et al. PD-1 inhibitor gastroenterocolitis: case series and appraisal of 'immunomodulatory gastroenterocolitis'. Histopathology 2017;70:558-567.

21. Beck KE, Blansfield JA, Tran KQ, et al. Enterocolitis in patients with cancer after antibody blockade of cytotoxic T-lymphocyte-associated antigen 4. J Clin Oncol 2006:24:2283-2289.

22. Nishimura $Y$, Yasuda M, Ocho K, et al. Severe gastritis after administration of nivolumab and ipilimumab. Case Rep Oncol 2018:11:549-556.

23. Johncilla M, Grover S, Zhang X, et al. Morphological spectrum of immune check-point inhibitor therapy-associated gastritis. Histopathology 2020;76:531-539.

24. Acero Brand FZ, Suter N, Adam JP, et al. Severe immune mucositis and esophagitis in metastatic squamous carcinoma of the larynx associated with pembrolizumab. J Immunother Cancer 2018;6:22.

25. Horisberger A, La Rosa S, Zurcher JP, et al. A severe case of refractory esophageal stenosis induced by nivolumab and responding to tocilizumab therapy. J Immunother Cancer 2018;6:156.

26. Niho S, Yoshida T, Akimoto T, et al. Randomized phase II study of chemoradiotherapy with cisplatin + S-1 versus cisplatin + pemetrexed for locally advanced non-squamous non-small cell lung cancer: SPECTRA study. Lung Cancer 2020;141:64-71.

27. Liu Q, Xia Y, Chen Y, et al. A study of concurrent chemoradiotherapy with weekly docetaxel and cisplatin for advanced esophageal squamous cell carcinoma with T4 and/or M1 lymph node metastasis or locoregional recurrence. Radiat Oncol 2020;15:75.

28. Brincker $\mathrm{H}$. Treatment of therapy-resistant acute myeloid leukaemia with 7 and 8 cytostatics. Scand J Haematol 1975;14:35-41.

29. Schadendorf D, Wolchok JD, Hodi FS, et al. Efficacy and safety outcomes in patients with advanced melanoma who discontinued treatment with 
nivolumab and ipilimumab because of adverse events: a pooled analysis of randomized phase II and III trials. J Clin Oncol 2017;35:3807-3814.

30. Postow MA, Sidlow R, Hellmann MD. Immune-related adverse events associated with immune checkpoint blockade. N Engl J Med 2018;378:158-168.

31. Bertrand $A$, Kostine $M$, Barnetche $T$, et al. Immune related adverse events associated with anti-CTLA-4 antibodies: systematic review and meta-analysis. BMC Med 2015;13:211.

32. Khoja L, Day D, Wei-Wu Chen T, et al. Tumour- and class-specific patterns of immune-related adverse events of immune checkpoint inhibitors: a systematic review. Ann Oncol 2017;28:2377-2385.

33. Horvat TZ, Adel NG, Dang TO, et al. Immune-related adverse events, need for systemic immunosuppression, and effects on survival and time to treatment failure in patients with melanoma treated with ipilimumab at Memorial Sloan Kettering Cancer Center. J Clin Oncol 2015;33:3193-3198.

34. Zhu AX, Finn RS, Edeline J, et al. Pembrolizumab in patients with advanced hepatocellular carcinoma previously treated with sorafenib (KEYNOTE-224): a non-randomised, open-label phase 2 trial. Lancet Oncol 2018;19:940-952.

35. Yoshida S, Miyamoto S, Naruse H, et al. Esophagitis in non-small cell lung carcinoma treatment caused by pembrolizumab. Am J Gastroenterol 2020:115:13

36. Richter JE, Rubenstein JH. Presentation and epidemiology of gastroesophageal reflux disease. Gastroenterology 2018;154:267-276.
37. Brown LF, Goldman H, Antonioli DA Intraepithelial eosinophils in endoscopic biopsies of adults with reflux esophagitis. Am J Surg Pathol 1984;8:899-905.

38. Patil PA, Zhang $X$. Pathologic manifestations of gastrointestinal and hepatobiliary injury in immune checkpoint inhibitor therapy [published online April 27, 2020]. Arch Pathol Lab Med, doi: 10.5858/arpa.20200070-RA

39. Wang DY, Kim DW, Shah NJ, et al. Clinical presentation of immune-related colitis associated with PD-1 inhibitor monotherapy (MONO) and combination PD-1/CTLA-4 inhibitors (COMBO) in melanoma [abstract] J Clin Oncol 2017;35(Suppl):Abstract 9566

40. Wang PF, Chen Y, Song SY, et al. Immune-related adverse events associated with anti-PD-1/PD-L1 treatment of malignancies: a meta-analysis. Front Pharmacol 2017;8:730.

41. Iwama S, De Remigis A, Callahan MK, et al. Pituitary expression of CTLA-4 mediates hypophysitis secondary to administration of CTLA-4 blocking antibody. Sci Transl Med 2014;6:230ra45.

42. Weber JS, Dummer R, de Pril V, et al. Patterns of onset and resolution of immune-related adverse events of special interest with ipilimumab: detailed safety analysis from a phase 3 trial in patients with advanced melanoma. Cancer 2013;119:1675-1682.

43. Kobayashi M, Yamaguchi O, Nagata K, et al. Acute hemorrhagic gastritis after nivolumab treatment. Gastrointest Endosc 2017;86: 915-916.

\section{FREE Patient Webinars}

National

Comprehensive

NCCN Cancer Network

\section{Register Now!}

\author{
NCCN.org/patientwebinars
}

People with cancer, their caregivers, and their families can join experts as they discuss treatment options and answer questions.

\section{Mantle Cell Lymphoma}

Wednesday, August 18, 2021 | 3:00 - 4:15 PM EDT

Wednesday, September 1, 2021 | 11:00 AM - 12:15 PM EDT

\section{Ovarian Cancer}

Thursday, August 19, $2021 \mid$ 4:15 - 5:30 PM EDT

Tuesday, August 31, 2021 | 12:15 - 1:30 PM EDT

\section{Coming soon!}

$\begin{array}{ll}>\text { Bladder Cancer } & >\text { Multiple Myeloma } \\ >\text { Non-Small Cell Lung Cancer } & >\text { Small Cell Lung Cancer } \\ >\text { Diffuse Large B-Cell Lymphoma } & \end{array}$

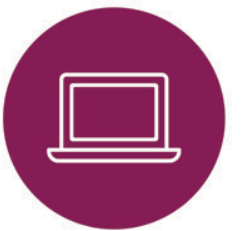

